\title{
Equidad de Género y las niñas víctimas de la trata: Otra forma de violencia
}

\author{
Trabajo de Reflexión y Planteamientos Metodológicos
}

Cristina Herrero-Villoria ${ }^{4}$, Antonia Picornell-Lucas ${ }^{5}$

\section{La Equidad de Género como herramienta para erradicar la violencia entre sexos}

Las situaciones de pobreza, el desequilibrio de poder entre sexos - la posición que ocupa la mujer en la sociedad-y la exclusión social, a la que se ve sometido el sexo femenino en la mayor parte de las sociedades modernas, propicia que miles de niños, niñas y adolescentes (NNA) deban sobreponerse a determinadas situaciones que ponen en peligro su etapa de crecimiento, considerada vital por las experiencias trascendentales que suponen para el ser humano.

A pesar del progreso experimentado en la mejora de las oportunidades esenciales para los NNA, diferentes estudios (Save the Children, 2015; UNICEF, 2015, 2017) demuestran que todavía hay muchos niños y niñas en el mundo que sufren privaciones $\mathrm{y}$ se encuentran en situaciones políticas, económicas y sociales desfavorables; existiendo disparidades entre los países y en el género, que afectan a su desarrollo y supervivencia.

Se genera así una importante brecha de desigualdad social entre niños y niñas en el momento de ejercer sus derechos. En el

${ }^{4}$ Máster en Criminalidad e Intervención Social en Menores por la Universidad de Málaga. Doctoranda en Estadística Multivariante Aplicada en la Universidad de Salamanca, Salamanca, España. E-mail: khrys@usal.es

5 Doctora en Ciencias de la Educación por la Universidad de Salamanca. Profesora Titular de Trabajo Social en la Universidad de Salamanca, Salamanca, España. E-mail: toi@usal.es 
último caso, esta inequidad se torna más sensible y aguda cuando se posiciona sobre las niñas y adolescentes, provocando su exposición a contextos de vulnerabilidad y exclusión social, con notables consecuencias para ellas. Fenómenos específicos como la prostitución infantil, la pornografía, la violencia machista, la trata para fines de explotación laboral o sexual, el matrimonio, la mutilación genital y/o el embarazo, entre otros; dando lugar a su vez a otros tipos de situaciones - violencia y malos tratos, discapacidad, migraciones, etc.- que delimitan sus opciones de progreso vital.

Una realidad vinculada a la construcción social de la identidad de género, que atribuye unas determinadas características a cada sexo: hombre y mujer, niño y niña. Así, se legitima una desigualdad de género, ya desde la infancia, convirtiendo al género en un factor más de discriminación. Una práctica que trasciende de las esferas públicas y privadas de la vida, limitando y poniendo en peligro el desarrollo de las niñas, sus libertades y oportunidades; dificultando su plena participación en la sociedad; además de atentar contra sus derechos y entorpecer su futuro y el de la comunidad en la que viven.

Un estudio realizado por Blum, Mmari y Moreau (2017), a través de entrevistas a niños de quince países del mundo -Ecuador, Bolivia, Bélgica, Escocia, Estados Unidos, Sudáfrica, Malawi, Kenya, RD Congo, Burkina Faso, Nigeria, Egipto, Vietnam, China e India- revela que los estereotipos femeninos están ya presentes en los niños y niñas de 10 años: las chicas son vulnerables, los chicos fuertes e independientes; las chicas son víctimas sexuales; los chicos, cazadores; etc. En definitiva, la inequidad de género persiste aún en los niños y niñas, quizá influenciados por la educación familiar que reciben en sus hogares, en contraste con las actividades que se ofrecen en la escuela. Para Binazzi (2016), solo una educación basada en derechos hará posible que las niñas puedan elegir libremente su futuro, evitando las relaciones asimétricas de poder.

En España, el Centro Reina Sofía sobre Adolescencia y Juventud, en el Barómetro Juventud y Género 2017 del ProyectoScopio, recogiendo las opiniones de los y las jóvenes, ofrece datos sobre las desigualdades de género, la violencia de género, los roles familiares, la feminidad y la masculinidad. Las conclusiones apuntan a que los jóvenes perciben que la 
mujer se encuentra en peor posición con respecto al hombre (56.4\%); con el aumento de la violencia machista (49\%) o con una gran discriminación salarial (75\%).

Para Amartya Sen (2016), aunque la falta de equidad de género no es homogénea en todo el mundo, hay desigualdad en la mortalidad - desnutrición y mínimos cuidados médicos-, en la natalidad —abortos sexoselectivos-, en las oportunidades básicas — acceso limitado a la educación, sanidad y participación en la comunidad-, en las oportunidades especiales —educación superior, preparación profesional一, en el ámbito profesional, en las posesiones y en el hogar.

Las dificultades que experimentan las niñas en el acceso a las oportunidades y a los recursos disponibles para su bienestar se revelan en las cifras aportadas por diversos organismos internacionales, a pesar de no estar suficientemente desagregados por género y edad y, en ocasiones, sin la posibilidad de disponer de datos reales de algunos países.

Existen indicadores de medición del desarrollo humano desde la perspectiva de género. Por ejemplo, Naciones Unidas utiliza el Índice de Desigualdad de Género (IDG) (Gender Inequality Index-GII), basado en el Indice de Desarrollo Humano (IDH). Este mide tres aspectos básicos del desarrollo: la salud reproductiva, el empoderamiento y la situación económica a fin de descubrir las desventajas que existen entre hombres y mujeres en su desarrollo humano. Pero, aunque en sus informes anuales revela las expectativas de escolarización de niños, no es posible localizar información detallada sobre las condiciones de vida de los NNA y visibilizar sus vulnerabilidades.

No obstante, el Grupo Interinstitucional y de Expertos sobre los indicadores de los Objetivos de Desarrollo Sostenible (Inter-Agency and Expert Group on Sustainable Development Goals Indicators, IAEG-SDG) ha elaborado un marco de indicadores mundiales para medir los progresos de los ODS (Naciones Unidas, A/RES/71/313). Pero el análisis de la equidad de género no se plantea solamente en el objetivo $\mathrm{n}^{\circ}$ 5: Lograr la igualdad de género y empoderar a todas las mujeres y niñas, sino también en el objetivo $\mathrm{n}^{\mathrm{o}}$ 4: Garantizar una educación inclusiva y equitativa de calidad, así como en el objetivo no1: Poner fin a la pobreza en todas sus formas y en todo el 
mundo. Y, aunque en el resto de los objetivos no se explicita la atención a las desigualdades de género, se contempla el 'sexo' como una posibilidad de desglose en todos los indicadores de evaluación.

También la UNESCO, consciente de la relación entre la igualdad de género y la educación, utiliza el Índice de Paridad de Género (IPG) para calcular el acceso relativo de los hombres y las mujeres a la educación - primaria, secundaria y superior- en aquellos países en vías de desarro1lo. A través del IPG se descubren datos como que 264 millones de niños no van a la escuela; que la brecha de desescolarización se produce en la educación primaria, donde 5 millones más de niñas que de niños no asisten a la escuela (9,7\% niñas y $8.1 \%$ niños); que la paridad en la educación primaria se ha alcanzado solo en el $66 \%$ de los países, aunque en menos de la mitad (45\%) se ha logrado en la secundaria y en el nivel terciario solo en el 4\% de los países (UNESCO, 2018). Estos análisis permiten desarrollar la idea de que hoy en día, a nivel mundial, todavía hay notables diferencias en el ámbito educativo en relación con el género.

Por su parte, UNICEF (2016) desarrolla estudios que permiten conocer que nueve de cada diez niñas viven en países de ingreso medio-bajo; que en algunos países del Asia Meridional y del Oriente Medio el peligro de morir antes de los 5 años es mayor en las niñas que en los niños, debido a la discriminación sexual; que una de cada cuatro adolescentes, entre quince y diecinueve años, muere por suicido en el Sudeste Asiático; que una de cada tres niñas, en los países donde se practica, son sometidas a la mutilación genital femenina; que tres de cada cuatro niñas están afectadas por el VIH en el África Subsahariana o que más de la mitad de las niñas de Somalia, Etiopía y Rwanda invierten catorce horas por semana en las tareas domésticas.

Para examinar con mayor detalle y poder determinar las líneas precisas de intervención ante estas situaciones que atentan contra el bienestar de la infancia y de la adolescencia, se están promoviendo metodologías estandarizadas de recogida de datos sobre su bienestar. A través de los indicadores $\mathrm{MICS}^{6}$ o de los del Report Card no 7, ambos de UNICEF;

${ }^{6}$ MICS: Mortalidad infantil; Nutrición; Salud infantil; Agua y saneamiento; Salud reproductiva; Desarrollo infantil; Educación; Protección infantil; VIH/SIDA, comportamiento sexual; Acceso a TICs; Bienestar subjetivo; Uso de tabaco y alcohol. 
de los indicadores de la Organización de Estados Iberoamericanos; de los indicadores de CEPAL para estimar la pobreza infantil; de los indicadores ISCI de International Society for Child Indicators o de los indicadores de The Annie E. Casey Foundation; entre otros. Y también a través de patrones concretos de los sistemas estadísticos nacionales sobre infancia y adolescencia, así como de otros instrumentos de medición diseñados por investigadores para valorar el bienestar infantil (ejs: Escala de percepción de indicadores de bienestar infantil en España -EPIBI; Sistema de indicadores sobre bienestar infantil en España-SIBI). A pesar de la proliferación de estos instrumentos para comprender la realidad infantil, podría decirse que han sido desarrollados de manera unilateral, sin una coordinación entre ellos; con gran disparidad en los datos y escasez de detalles en relación con la situación específica de las niñas y las adolescentes.

La comunidad mundial está experimentando grandes avances en la reducción de las prácticas discriminatorias y de exclusión contra las niñas. Las Naciones Unidas, en colaboración con otras organizaciones comunitarias y con los gobiernos, está abordando la desigualdad de género mediante "el empoderamiento de las mujeres como agentes del cambio y líderes de los procesos de desarrollo que influyen en sus vidas”, como señala la Estrategia sobre igualdad de género del Programa de las Naciones Unidas para el Desarrollo, 2014-2017 (DP/2014/4).

La igualdad de género es un tema de particular preocupación para diversos organismos internacionales, entre ellos la ONU. En la Agenda 2030 recoge la igualdad entre los géneros y el empoderamiento de todas las mujeres y niñas como objetivo $n^{\circ} 5$, a la vez que especifica que este objetivo, y la perspectiva de género, ha de ser transversal al resto de los objetivos, metas e indicadores de los Objetivos de Desarrollo Sostenible, para "identificar, producir y difundir información que refleje las realidades de las vidas de las mujeres y los hombres" (ONU Mujeres, 2017:5). De esta manera, se está colocando a la mujer y a la niña en el centro del desarrollo sostenible a la vez que se defienden sus derechos.

Este mismo enfoque integrado de la perspectiva de género ya fue reconocido y recogido por la OIT, que puso en marcha en el año 2000 el Plan de Acción para la Igualdad de Género y la Transversalización de la Perspecti- 
va de Género para garantizar mecanismos de análisis de las dificultades y oportunidades en el mercado de trabajo. Aunque, en el último estudio publicado, afirma que "las iniciativas actuales de los principales actores del mercado de trabajo para reducir la desigualdad en el mercado laboral entre mujeres y hombres no son suficientes" y es obligatorio redoblar los esfuerzos (OIT, 2018:13).

La perspectiva de género tuvo sus inicios en los años 70, con la celebración en México de la primera Conferencia Mundial del Año Internacional de la Mujer, organizada por la ONU. Aunque fue la Declaración y la Plataforma de Acción de Beijing (1995), el documento que reafirmó el compromiso político de los gobiernos a trabajar contra la discriminación de género y a favor de la igualdad en todos los ámbitos de la vida. Y una de las esferas de especial preocupación eran las niñas, "consideradas inferiores y [a las que] se les enseña a ponerse siempre en último lugar, con lo que se les quita el sentido de su propia dignidad" (ONU Mujeres, 2014:189). En este contexto, plantea 9 objetivos estratégicos para conseguir avanzar en el reconocimiento de los derechos de las niñas: 1) eliminar todas las formas de discriminación contra la niña, 2) eliminar las prácticas culturales perjudiciales, 3) intensificar la conciencia de las necesidades y potencial de la niña, 4) eliminar la discriminación en la educación, la salud y la nutrición, 5) eliminar la explotación económica, 6) erradicar la violencia contra las niñas, 7) concienciar a las niñas sobre su participación en la sociedad, 8) fortalecer la función familiar; así como una serie de medidas para orientar a los gobiernos en su cumplimiento. Tras 20 años desde su aprobación, se han ido añadiendo otros problemas, como la trata de personas, la corrupción o la droga, que afecta de manera especial a las mujeres y a las niñas, según la propia Declaración.

\section{La perspectiva de género en la lucha contra la trata de niñas con fines de explotación sexual}

La transversalidad en la dimensión del género debería consolidarse como cultura política en la atención y protección social de todos los NNA. UNICEF (2016) confirma que las niñas siguen en desventaja y que es preciso priorizar servicios e infraestructuras que evalúen las vulnerabilidades de las niñas y remuevan barreras de su empoderamiento. 
La consideración del género femenino como artículo de compra y venta, sin derechos y sometida de forma constante a la potestad y deseo sexual del hombre (Viveros, 2008), junto al sistema patriarcal en el que todavía se educa a gran parte de la población mundial, propicia que el crimen de la trata de seres humanos con fines de explotación sexual (TSHFS) englobe a un volumen elevado de féminas (Cortés y otros, 2011). Provocando que ambos sexos estén posicionados en lugares opuestos de la balanza: oferta (niñas) frente a demanda (hombres). Según los últimos datos manejados por la Oficina de las Naciones Unidas contra la Droga y el Delito (UNODC, por sus siglas en inglés), en el año 2016, el 71\% de las víctimas de explotación sexual pertenecen al género femenino; en concreto, el $28 \%$ de estas eran NNA, 8\% niños y 20\% niñas. Según la OIT (2017c), el 70\% de las víctimas fueron explotadas en Asia y la región del Pacífico, Europa y Asia Central (14\%), África (8\%), las Américas (4\%) y en la Liga Árabe (Medio Oriente y Magreb) (1\%).

En Europa, y también en España, está presente el delito de trata. Según la Oficina Estadística Comunitaria Eurostat (2015), en los 28 Estados miembros de la Unión Europea, entre los años 2010 y 2012, el último periodo del que se disponen datos, se registraron como víctimas de la trata 30.146 personas —un 28\% más que en el trienio anterior-; el 95\% de las víctimas de explotación sexual eran del género femenino y más de 1.000 NNA fueron objeto de explotación sexual. En España se contabilizaron 1.605 víctimas de explotación sexual —identificadas y estimadas- (1.472 mujeres y 133 hombres), aunque no se ofrecieron datos desagregados por edad. No obstante, el Informe señala que "la tasa de prevalencia de las víctimas registradas en Francia, Alemania, Reino Unido y España ha sido relativamente baja a pesar de los altos números absolutos de víctimas registradas en estos países" (pg. 34).

Considerando todos estos datos y manifestaciones, se puede debatir acerca de la existencia o no de un consenso mundial, o europeo, sobre el fenómeno de la trata de NNA. A pesar de ser una situación de extrema gravedad, los datos publicados son dispares e inconexos y esta dispersión impacta en las respuestas de los gobiernos, de las organizaciones internacionales y de la sociedad civil. En otra dimensión, se trata de una realidad que insiste en considerar al patriarcado como un modelo social todavía 
influyente, a pesar de la evolución de las sociedades. Los ideales intrínsecos a la organización social hetero-hegemónica persisten y los edictos hacia las mujeres continúan presentes (sumisión, reproducción, etc.), siendo rechazadas por la sociedad si se apartan de lo 'normalmente' establecido. Por el contrario, el hombre, desde una posición más privilegiada, se 'dedica' al ocio y al placer que le proporciona el género femenino (Díaz, 2014).

Es importante destacar que la Convención sobre la Eliminación de Todas las Formas de Discriminación contra la Mujer (CEDAW, por sus siglas en inglés, 1979) establece en su Art.2.b que la TSHFS debe ser considerada una inexorable expresión de violencia de género, y solicita a los Estados Partes se tomen "todas las medidas apropiadas, incluso de carácter legislativo, para suprimir todas las formas de trata de mujeres y explotación de la prostitución de la mujer" (Art.6).

Específicamente, la Unión Europea está abordando el tema por medio de instrumentos normativos, con especial atención a la dimensión de género, como la Convención contra el Tráfico de Seres Humanos (2005), la Directiva 2011/36/EU relativa a la Prevención y Lucha contra la Trata de Seres Humanos y la Protección de sus Victimas o la creación de un grupo de expertos (GRETA), en el año 2008, para evaluar el cumplimiento de la Convención y proponer buenas prácticas, priorizando cualquier acción contra el tráfico de NNA. Estas medidas reconocen la necesidad de establecer medidas específicas de ayuda para los niños y niñas debido a sus circunstancias de especial vulnerabilidad. Han de tener en cuenta el superior interés del niño y por ello se despliegan en consonancia con la Estrategia del Consejo de Europa para los Derechos de los Niños (2016-2021), que contempla como una de sus áreas prioritarias 'una vida libre de violencia', donde se incluye el abuso sexual y la explotación.

Todo ello se ha materializado en la Estrategia de la UE para la erradicación de la trata de seres bumanos (2012-2016), dentro de la cual merece una atención especial la alusión a la necesidad de mejorar la coordinación y la coherencia de las políticas. Se constata así un mayor esfuerzo a fin de responder eficazmente a la detección y protección de las víctimas, perseguir más activamente a los traficantes y reforzar la prevención; prioridades en las que debe centrar sus actuaciones la UE para defender los derechos de 
las víctimas de la trata de seres humanos. Precisamente, dentro de esta Estrategia de la UE, en el año 2013 se aprueba un documento que recoge los derechos de las víctimas de la TSH, entre los que se encuentran, además de la asistencia y apoyo, la indemnización y la inserción educativa y/o laboral, entre otros relacionados con la concesión de un permiso de residencia y el retorno a su país (Comisión Europea, 2013).

Todas estas medidas normativas y procedimentales, a pesar de permitir una mejor coordinación entre las regiones, no alcanzan a proporcionar una respuesta eficaz a las víctimas y, más concretamente a los niños, niñas y adolescentes. Un estudio de la OIT (2017d), sobre el trabajo infantil en el mundo, estima que en América, una de cada veinte niñas - y niños- se encuentra en situación de trabajo infantil (5,3\%), una proporción superior a las zonas de Europa y Asia Central $(4,1 \%)$ o a los Estados árabes $(2,9)$. En cuanto a las niñas, entre los años 2012-2016, hasta 64 millones realizaron algún tipo de trabajo infantil en el mundo (OIT, 2017b), "responsabilidad desproporcionada” frente a sus iguales varones (OIT, 2017a:7), viéndose obligadas a llevar una doble carga de trabajo o a dejar al margen su promoción educativa o profesional para sostener económicamente a su entorno familiar dedicándose al cuidado de los menores de edad, de la casa y de otras personas dependientes.

Esta separación de roles ocasiona una "gran desigualdad de oportunidades en el acceso a los recursos materiales y derechos políticos" (Castellanos y Ranea, 2013:54). Además, es interiorizada desde edades muy tempranas, propiciando que algunas sociedades toleren e incluso normalicen el trabajo en régimen de servidumbre, que las tareas domésticas son específicas de las mujeres y, entre otras cuestiones, la cosificación del cuerpo femenino como "objeto al servicio de la mayor satisfacción de los hombres", reforzando aún más — si cabe- la discriminación por razón de sexo, caracterizada por ser "intencional, no neutra y continuamente enmascarada" (Ruiz y otros, 2005:22).

Los conflictos bélicos (1000 millones de niños viven en países que han sufrido esta situación), los desastres naturales, las epidemias, las desigualdades en el derecho a la educación o la imposibilidad de acceder, las legislaciones y políticas nacionales que restringen la igualdad de género, las 
prácticas culturales, la pobreza de las familias, la violencia... (Plan Internacional, 2015) produce fragilidad en los NNA. En la búsqueda de una vida mejor se sitúan, de forma involuntaria, en escenarios de riesgo en el que, como en el caso de la Trata de Seres Humanos (TSH), las niñas son explotadas sexualmente. Esto se traduce en una fuerte representación del género femenino en las migraciones contemporáneas internacionales; favoreciendo el crecimiento global de la industria del sexo tanto en los países de destino como en los de origen (turismo sexual). En el caso de España, la migración feminizada proviene de América Latina: Ecuador, Colombia, Bolivia, Perú, Argentina y, especialmente, Brasil, el único país en el que el porcentaje de mujeres emigrantes supera el $64 \%$ con respecto a los hombres. (Castellanos y Ranea, 2013). Esta demanda de mujeres para el mercado de la prostitución supone el principal incentivo de las redes criminales de la trata de seres humanos, quienes parten con ventaja ante las restrictivas políticas de movimiento y migración que hay en las fronteras. Para estos mismos autores, esto provoca que una parte del flujo migratorio, que no posee los medios necesarios para pasar dichos controles, deposite su confianza en las entidades delictivas bajo la promesa de un futuro mejor.

Tal incidencia y complejidad del crimen de la TSH en las distintas esferas de la vida de las niñas — psicológica, sexual, económica, educativa, etc.- plantea la necesidad de intervenir desde una metodología de atención integral. Sin generar desequilibrios como la revictimización en el ámbito institucional, es decir, por la existencia de obstáculos que impidan el acceso justo de las niñas al sistema de justicia y/o a los servicios de atención y protección necesarios para su plena reinserción social. Como afirma Alcáza-Campos (2013), la práctica profesional —en concreto se refiere al trabajo social- ha de prestar atención a la inercia de las instituciones públicas, que contradice los principios de la profesión y subordina a las niñas. Esta reflexión hará posible la erradicación de prácticas profesionales que generan desigualdades de género y exclusión social de las niñas, impulsando el necesario cambio estructural que demandan las situaciones de discriminación en las que viven las niñas.

Reducir las inequidades de género supone romper con los modelos de masculinidad y con las relaciones de poder-sumisión entre hombres y mujeres - niños-, y con ello, entre otras cuestiones, a desanclar el fenómeno 
de la TSHFS al paso del tiempo (Pérez, 2013). La eliminación de los prejuicios sexistas y la deslegitimación de ciertas prácticas abusivas, de subordinación y opresión aumentará los apoyos en la lucha por la igualdad real; intensificando unas condiciones de vida dignas para que todas las niñas $-\mathrm{y}$ mujeres - puedan ejercer sus derechos y desarrollarse libre y plenamente.

\section{Conclusiones}

Como puede advertirse, para combatir esta forma de "violencia remunerada” y cualquier otro tipo de trasgresión, es preciso un intenso y extenso trabajo preventivo, de intervención y punitivo, con soporte estructural en la perspectiva de género y, por supuesto, en el enfoque de derechos humanos. Y aunque la TSHFS está adquiriendo una mayor institucionalización, mediante la formación y desarrollo de normativas y metodologías comunes de intervención, las cuales han mejorado la investigación de los factores que llevan a esta actividad delictiva, es necesario un mayor esfuerzo en todas las regiones del mundo.

Es preciso trabajar en la construcción de sociedades igualitarias, apoyadas en el discurso coherente sobre la igualdad entre géneros y las prácticas reales que mantienen y fomentan los estereotipos que perpetúan el patriarcado; como, por ejemplo, la divergencia de opiniones respecto al ejercicio de la prostitución - forma cardinal de explotación sexual infantil y adulta - Ser congruentes exige romper el silencio cómplice y comprometerse de forma activa en la lucha para erradicar todas las formas de violencia contra las niñas - y mujeres-. Ello precisa de una educación basada en la igualdad de género, en el respeto al otro y en la visión del género femenino como sujeto de derechos.

Es prioritario combatir el problema de la pobreza feminizada - principal causa de la trata- mediante, entre otras cuestiones, el despliegue de acciones que faciliten el acceso de las niñas al sistema educativo. Una mayor formación supondrá mayores dificultades para que puedan ser manipuladas y capturadas por las redes criminales. Una educación que también ha de implicar a los varones, desde edades tempranas, en la lucha contra la trata de manera bilateral, para que puedan crecer asumiendo y reconociendo la igualdad entre sexos y el espacio del género femenino en la sociedad. 
A su vez, para fortalecer la ciudadanía de las niñas, transformar las prácticas nocivas, como la TSHFS, entre otras, y otorgarles el lugar que les corresponde, es necesario "incorporar una perspectiva de género en todas las políticas y programas de forma que antes de adoptar cualquier decisión se analice de qué forma afectará a la niña y al niño, respectivamente" (ONU Mujeres, 2014: 192). Urge un cambio cultural en la deconstrucción de los estereotipos de género, con especial referencia al enfoque de derechos de la infancia. 


\section{Referencias bibliográficas}

Alcázar-Campos, A. (2013). Social Work with Female Victims of Gender Violence: Analysis of an Experience in Southern Spain from a Feminist Perspective. Affilia. Women and Social Work, 28 (4), 366-378. DOI: 10.1177/0886109913504722.

Binazzi, A. (2016). The role of formal and non-formal education for children's empowerment and as a prevention tool from violence. Comparative Cultural Studies: European and Latin America Perspectives, 2, 77-87. DOI: 10.13128/ccselap-19999.

Blum, R.; Mmari, K. y Moreau, C. (2017). It Begins at 10: How Gender Expectations Shape Early Adolescence Around the World. Journal of Adolescente Health, 61(4), supplement, S3-S4. DOI: http://dx.doi. org/10.1016/j.jadohealth.2017.07.009

Castellanos, E. y Ranea, B. (2013): Explotación sexual de mujeres. Investigación sobre prostitución y trata de mujeres. Madrid: Asociación de Promoción de Servicios Sociales (APROSERS).

Comisión Europea (2013). Los derechos de las víctimas de la trata de seres bumanos en la UE. Luxemburgo: Oficina de Publicaciones de la Unión Europea.

Cortés, J. del P.; Becerra, A.; López, S. y Quintero, L. (2011): ¿Cuál es el problema de la trata de personas?: revisión de las posturas teóricas desde las que se aborda la trata. Nova et Vétera, 20 (64), 105-120.

Díaz R, A. (2014): Guía para la comunicación de la violencia de género: Manual de intervención ante la trata con fines de explotación sexual. Jaén: Dirección General de Violencia de Género y Asistencia a Víctimas.

OIT_ Organización Internacional del Trabajo (2017a). Poner fin al trabajo infantil a más tardar en 2025: Análisis de politicas y programas. Resumen ejecutivo. Ginebra: Oficina Internacional del Trabajo. 
OIT- Organización Internacional del trabajo (2017b). Trabajo infantil: Estimaciones y tendencias mundiales, 2012-2016. Ginebra: Oficina Internacional del Trabajo.

OIT-Organización Internacional del Trabajo (2017c). Global Estimates of Modern Slavery: forced labour and forced marriage. Geneve: International Labour Office (ILO).

OIT- Organización Internacional del Trabajo (2017d). Global Estimates of Modern Slavery: forced labour and forced marriage. Regional brief for Americas. Geneve: International Labour Office (ILO).

OIT- Organización Internacional del Trabajo (2018). Perspectivas sociales $y$ del empleo en el mundo. Tendencias del empleo femenino. OIT: Ginebra.

ONU Mujeres (2014; 1ª 1995). Declaración y Plataforma de Acción de Beijing. Nueva York.

ONU Mujeres (2017). Transversalización de género en los Objetivos de Desarrollo Sostenible-Agenda 2030. ONU: Nueva York.

Pérez, S. (2013). Informe: estudo explotario da trata de persoas en Galicia. España: Junta de Galicia.

Plan Internacional (2015). El estado mundial de las niñas: Los asuntos pendientes de los derechos de las niñas. España: Plan Internacional.

Ruiz, B.; Martínez, E.; Martínez, A.; Bascuñana, C. y Cutrín M. (2005): Esclavas en tierra de nadie. Acercándonos a las victimas de la trata de mujeres. Madrid: Red Acoge.

Save the Children (2015). La lotería del nacimiento. Démosle a toda la infancia la misma oportunidad de supervivencia. Londres; Reino Unido.

Sen, A. (2016). La desigualdad de género. La misoginia como problema de salud pública. Consciencia y Diálogo, 6, enero-diciembre, 213-219.

UNESCO (2018). Global Education monitoring report gender review. Meeting our commitments to gender equality in education. UNESCO: París, Francia. 
UNODC. Oficina de las Naciones Unidas contra la Droga y el Delito. (2016): Global Report on Trafficking in Persons. United Nations: New York.

UNICEF (2015). El compromiso con la supervivencia infantil: una promesa renovada. UNICEF: Nueva York.

UNICEF (2016). Harnessing the Power of Data for Girls Taking stock and looking abead to 2030. UNICEF: New York.

Viveros, M. (2008; 2a ed.): “Teorías feministas y estudios sobre varones y masculinidades. Dilemas y desafíos recientes”. En J. Ramírez y G. Uribe (Eds). Masculinidades: El juego de género de los hombres en el que participan las mujeres. Madrid: Plaza y Valdés. 